\title{
Reconstructive and Restorative Interventions at the Proximal End of the Thigh and Pelvic Bones in Destructive Pathological Dislocation of the Hip in Children After Hematogenous Osteomyelitis
}

\author{
Khojaakhmed Shaykhislamovich Alpysbaev \\ Head of the Department of Hip Pathology of the Republican Center for Pediatric Orthopedics of the Ministry of \\ Health of the Republic of Uzbekistan \\ Corresponding author email:xojaahmed@mail.ru
}

\author{
Akhror Makhmutovich Djuraev \\ Chief of the Department of Pediatric Orthopedics of the Republican Specialized Scientific and Practical Medical \\ Center of Traumatology and Orthopedics of the Ministry of Health of the Republic of Uzbekistan \\ Elyar Abduvalievich Tapilov \\ Department of Pathology of the Hip Joint of the Republican Center for Pediatric Orthopedics of the Ministry of \\ Health of the Republic of Uzbekistan
}

\begin{abstract}
It is generally known that children tend to get injured more easily than adults. Moreover, these injuries they get in growing period may leave for long in the forms of disability. This paper is about reconstructive and restorative interventions at the proximal end of the thigh and pelvic bones in destructive pathological dislocation of the hip in children after hematogenous osteomyelitis. After hematogenous osteomyelitis of the proximal end of the femur, destruction of the head and neck of the femur is often observed, up to their destruction. The optimal age for surgical treatment of pathological dislocation of the hip, according to our data, is 4-5 years of age of the child, because by this time the process of ossification of the structures of the hip joint ends in most patients, and early surgical intervention often causes severe secondary deformities, up to their destruction.

Keywords---cervico-shaft angle, hip joint, osteomyelitis, pathological hip dislocation, treatment
\end{abstract}

\section{Introduction}

The relevance of the problem: Pathological dislocation of the hip (PDH) is a violation of the ratios in the hip joint due to severely hematogenous meta-epiphyseal osteomyelitis, which is based on the destruction of the fibrous capsule of the joint, the round ligament of the head, and the proximal meta-epiphyseal zone of the femur by the purulent-necrotic process. Acute hematogenous osteomyelitis accounts for 6-12.2\% of purulent diseases and affects long tubular bones in 79.1-88.7\% of cases (Shamsiev, 2018). Most often in patients with this pathology, the proximal meta-epiphysis of the femur is affected. Acute hematogenous meta-epiphyseal osteomyelitis of the proximal femur occurs in 24.6-50\%, cases (Shikhabudinova et al., 2000), accounts for $2.6-11 \%$ of all surgical and $6.6-30 \%$ of purulent diseases of childhood (Akzhigitov, 1998; Korovin et al., 2018), while the neonatal period accounts for up to $27.4 \%$ of cases of pathology (Shamsiev et al., 2018).

According to Labuzov et al. (2017), from $75 \%$ to $100 \%$ of children with hematogenous osteomyelitis is admitted to the hospital late, which contributes to the development of orthopedic complications that develop in $22-71.2 \%$ of children and 16.2-53.7\% of patients lead to early disability (Garkavenko \& Pozdeev, 2011; Nikolaev, 2005; Garkavenko \& Pozdeev, 2013; Skvortsov et al., 2011; Sokolovsky \& Sokolovsky, 1997; Choi et al., 1990). According to the literature, in $43-50 \%$ of cases, the consequences of septic coxitis are deformity of the proximal femur, dysplasia of the acetabulum, and impaired articular relationships (Krys'-Pugach, 2008; Sitkovsky \& 
Kukuruza, 1975). Surgical treatment of patients with complete defect of the pineal gland, complete defect of the head and neck of the femur, or premature closure of the growth zone of the greater trochanter is an extremely difficult problem in pediatric orthopedics and up to $71.2 \%$ of cases gives unfavorable results in the form of joint instability, coxarthrosis with limb shortening (Garkavenko \& Pozdeev, 2011; Cheng et al., 1995; Di Gennaro et al., 2003; Forlin \& Milani, 2008). Rehabilitation treatment of especially severe PVB in children with a complete defect of the femoral head and neck (Forlin \& Milani, 2008), as well as with a defect in the head and neck of the femur with damage or premature closure of the growth zone is the most difficult task of modern orthopedics (Sitkovsky \& Kukuruza, 1975). Modern surgical treatment of residual deformities of purulent coxitis includes all the latest reconstructive and restorative interventions developed at the proximal end of the thigh and pelvic bones (Garkavenko \& Pozdeev, 2011; Teplenky et al., 2016). In the surgical treatment of pathological dislocation, the primary task is to restore the support function of the limb while maintaining mobility in the joint, followed by solving the problem of eliminating secondary deformities and equalizing the length of the lower limbs (Garkavenko \& Pozdeev, 2013). In this regard, we have developed a new method of surgical treatment of destructive pathological dislocation of the hip, aimed at increasing the efficiency of open reduction of the remaining stump of the head and neck by restoring optimal anatomical and functional relationships in the hip joint.

\section{The objective of the study}

To improve the results of treatment of destructive pathological hip dislocation by developing methods of surgical treatment.

\section{Materials and Methods}

We observed 74 patients with destructive pathological dislocation of the hip at the age of 3 to 14 years. Patients were examined using clinical, radiographic, MRI, and MSCT methods of investigation. In all patients, deformity of the proximal end of the femur: a decrease or increase in the cervico-diaphyseal angle of the femur, shortening of the femoral neck, high standing of the greater trochanter, and a negative value of the articulotrochanter distance. When $\mathrm{X}$-ray examination of children, X-ray of the hip joints was performed in the anteroposterior projection with the middle position of the hips and with internal rotation, as well as in the Lauenstein position. Radiographs were used to measure parameters characterizing the angular values of the hip joint and proximal femur, the angle of the vertical slope of the depression, the angle of vertical correspondence, the acetabular angle, the cervico-diaphyseal angle, and the angle of anteversion, the Alsberg angle, and the coefficient of bone coverage. In most patients, according to the study methods, the proximal ends of the femurs consisted of the residual stump of the head and neck or large trochanters (Morrey \& Peterson, 1975; Karmazyn, 2010).

\section{The main findings and results}

The planning of surgical intervention in patients was based on the assessment of disturbances in the anatomical relationships in the hip joint, its function, disturbances in the spatial orientation of the bones that form the joint, as well as disturbances in the size, shape, and integrity of bones and was carried out taking into account the identified symptom complexes and the patient's age. The requirement for reconstructive surgery was the preservation of cartilaginous structures, growth zones, and sufficient blood supply to the hip joint. The final nature and extent of the intervention were determined intraoperatively, after a visual assessment of the components of the joint and its stability (Kardina et al., 2020; Suwananta et al., 2021). Much attention was paid to predicting changes in the ratios in the joint after the intervention, taking into account the remodeling of its components in the process of the child's growth. the process of ossification of the structures of the hip joint, when secondary deformities have not yet formed and the regeneration processes are well expressed. The indication for the use of corrective osteotomies is a combination of clinical and radiological data - lameness, faulty positioning of the lower extremities, significant limitation of hip abduction, weakness of the gluteal muscles, impaired relationships in the hip joint, varus deformity of the femoral neck, high position of the greater trochanter and shortening of the lower limb. The decisions were made strictly individually; for each patient, the amount of the proposed correction was determined, taking into account the already occurring irreversible secondary changes (structural scoliosis, pelvic misalignment) and age.

Clinical example No. 1. B-oh.: Z.I. No. i / b 669/181. He was admitted to the department with complaints of lameness in the right leg, shortening of the right lower limb, and restriction of abduction to the right of the hip joint. From the anamnesis: on the 6th day after birth, the child's body temperature rose (380-390.). The parents noticed 
asymmetry in the hip joints and skin folds on the thighs. Subsequently, these symptoms were joined by a sharp restriction of abduction and extension in the right hip joint. After the research methods were carried out, the doctor was diagnosed: Pathological destructive dislocation of the right femur. Flexion-conducting contracture of the right hip joint. Figure 1(a).

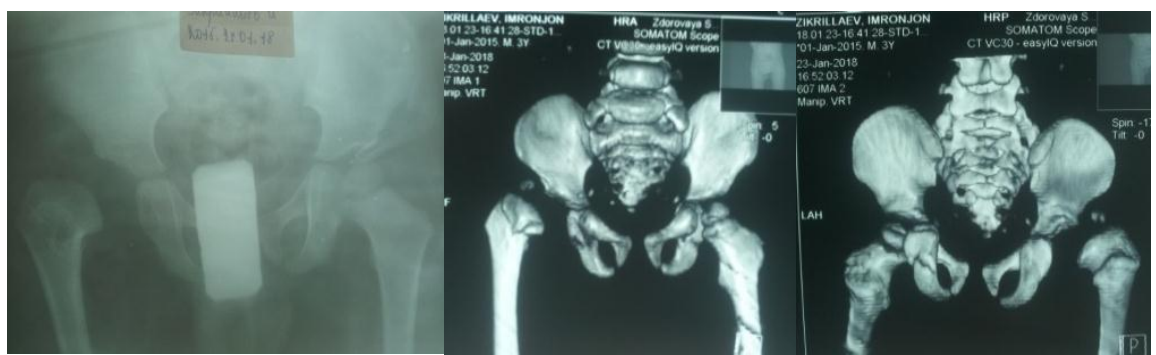

Figure 1(a). X-ray and MSCT picture of pathological destructive dislocation of the right hip

An open reduction of the stump of the head and neck of the right femur was performed with an intertrochanteric torsiondevarizing and "lengthening" neck osteotomy of the femur and rotational osteotomy of the ilium according to Salter. Rice. No. 1 ( b, c, d)

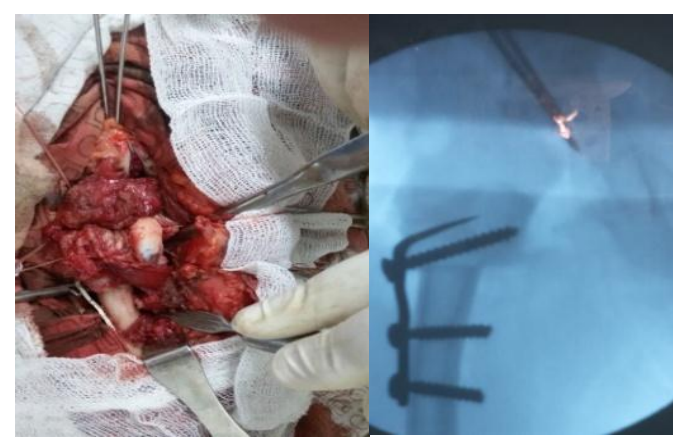

Figure 1(b)
Figure 1(c)

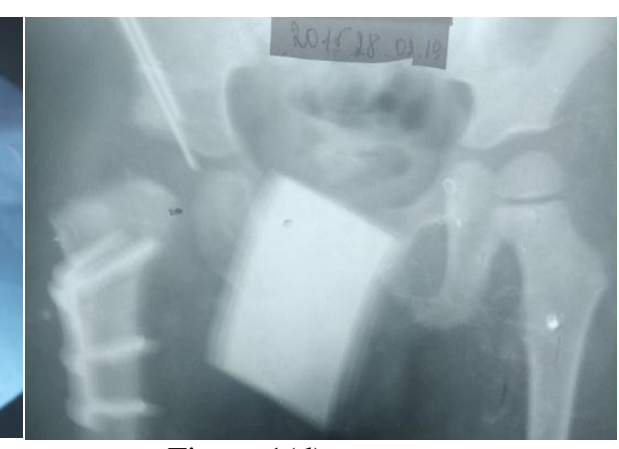

Figure 1(d)

Figure 1(b). Intraoperative picture: the remaining stump of the femoral head is deformed, deformity in the form of "auricle". Osteosynthesis of bone fragments with a Blount plate and 3 screws. ShDU within 1100-1150.

Figure 1(c). Electron-optical converter (EOP) control during the operation: the remaining stump of the femoral head is located in the cavity and is completely covered with the roof of the acetabulum due to the roof slope laterally and downward with insertion into the grooved groove of the autograft. Osteosynthesis of bone fragments with a Blount plate and 3 screws. SDA within 1100-1150. After the operation, a hip plaster cast was applied. The patient received antibacterial and symptomatic treatment. After the operation, the wound healed primarily, the sutures were removed 16-18 days after the operation. 1.5 months after the operation, the hip plaster cast was removed and a plaster cast "boot with a deratator" was applied to the right lower limb for 2-2.5 months. The patient received 2 courses of rehabilitation measures with the inclusion of exercise therapy of the hip joints.

Figure 1(d). The result is 7 months after the surgical treatment, before the removal of the hardware from the wing of the ilium and the proximal femur: the head of the femur is in the cavity and is completely covered with the roof of the acetabulum. Osteosynthesis of bone fragments with a Blount plate and 3 screws. ShDU within 1200. Complete consolidation of bone fragments.

7 months after the surgical treatment under combined anesthesia, the metal structures were removed from the proximal end of the right femur and the wing of the ilium. 4 months after the removal of the metal structures, the patient was allowed to walk. Currently, the patient walks on his own, chromate has disappeared, movements in the hip joints: flexion - $110^{\circ} / 0^{\circ} / 120^{0}$; abduction $-50^{\circ} / 0^{\circ} / 60^{\circ}$; internal rotation- $20^{\circ} / 0^{\circ} / 40^{\circ}$; external rotation $-25^{\circ} / 0^{0} / 45^{0}$. Trendelenburg's symptom is negative. The greater trochanter of the right femur does not protrude and is located on the Roser-Nelaton line. The length of the lower limbs is the same (Lew \& Waldvogel, 2004; Parsons \& Strauss, 2004). 
Clinical example No. 2. Patient M., 5 years old, No. and / b No. 2554. On September 18, 2007, she was admitted to the Department of Pathology of the Hip Joints with a diagnosis of pathological destructive dislocation of the hips. Objectively, all signs of bilateral hip dislocation were observed - lameness when walking in both directions "duck gait", a sharp restriction of movements in the hip joints: flexion - 80/0 $/ 90^{0}$; abduction - $10^{\circ} / 0^{0} / 20^{0}$; internal rotation $10^{\circ} / 0^{0 / 1} 15^{0}$; external rotation $-20^{0} / 0^{0} 25^{0}$. Trendelenburg's symptom is sharply positive. The greater trochanter of the femur protrudes and is above the Roser-Nelaton line. The length of the lower limbs is the same. The plain X-ray showed a pathological destructive dislocation of both thighs with the formation of a "saddle" deformity of the remaining stump of the head and neck, varus deformity and shortening of the femoral neck "coxabreva". Figure 2(a).
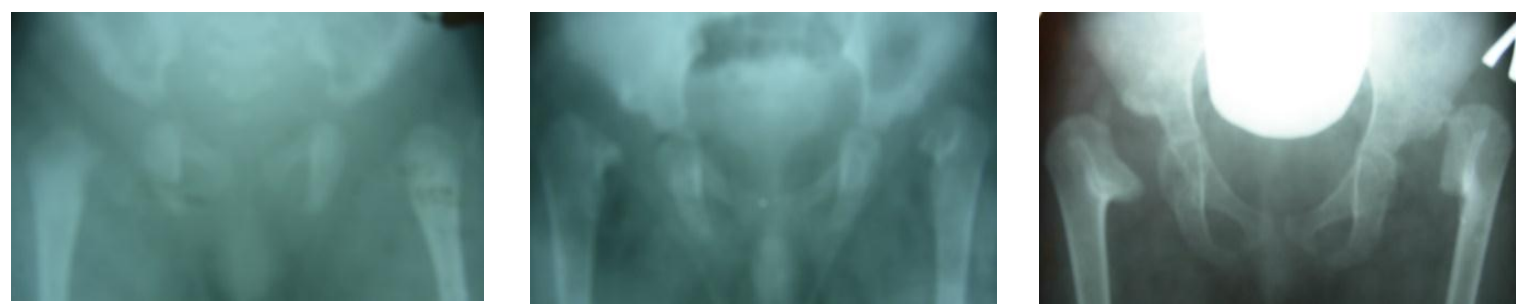

Figure 2(a). Radiographs of the hip joints produced in dynamics: Pathological destructive dislocation of the hips with saddle deformity of the remaining stump of the head and neck, varus deformity, and shortening of the neck of the femoral bones "coxabreva".

At the first stage, the patient on September 24, 2007, under general anesthesia, underwent open reduction of the remaining stump of the head and neck of the right femur with modulating resection of the last and intertrochanteric shortening detorsion-varizing-"lengthening" neck by osteotomy according to the above-described method. Figure 2 (b). After the operation, a hip plaster cast was applied. 1.5 months after the operation, the hip plaster cast was removed and a plaster cast "boot with a deratator" was applied to the right lower limb for 2-2.5 months. The patient received the first course of rehabilitation measures. In 4.5-5 months after the operation, the patient received the II course of rehabilitation measures with the inclusion of Physical Therapy (exercise therapy) of the hip joints. On March 26, 2008, under general anesthesia, an open reduction of the stump of the head and neck of the left femur was performed with modulating resection of the last and intertrochanteric shortening detorsion-varizing- "lengthening" neck osteotomy according to the above-described technique Figure 2(b).

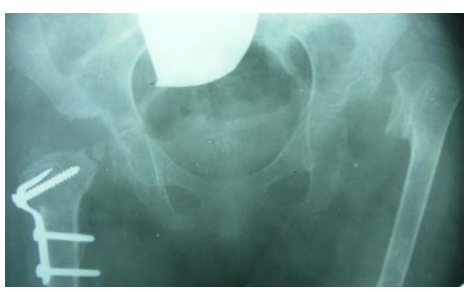

Figure 2(b)

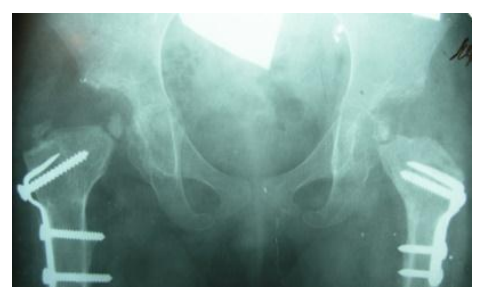

Figure 2(c)

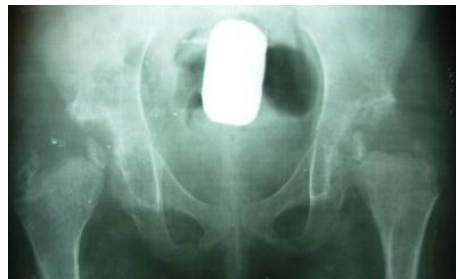

Figure 2(d)

Figure 2(b). SPO. Open reduction of the stump of the head and neck of the right femur with an intertrochanteric shortening detorsion-varizing-"lengthening" neck osteotomy and modulating resection of the latter. The stump of the head and neck of the right femur with the preserved growth zone is located in the cavity, centered on the bottom of the acetabulum; the fragments of the femur are fixed with a Blount-type metal plate and 3 screws.

Figure 2(c). SPO. Open reduction of the remaining stump of the head and neck of the left femur with an intertrochanteric shortening detorsion-varizing-lengthening osteotomy and modulating resection of the latter. The stump of the head and neck of the left femur with a preserved growth zone is located in the cavity, centered on the bottom of the acetabulum; the bones were fixed with a Blount-type metal plate and 3 screws. On February 12, 2009, under combined anesthesia, the metal structures were removed from the proximal end of both femurs. 3 months after the removal of the metal structures, the patient was allowed to walk with crutches. Figure 2(d).

Figure 2(d). SPO. The result of surgical treatment: the stumps of the head and neck of the femur are located in the cavities, centered on the bottom of the acetabulum. The cervico-diphyseal angle is within the normal range: 1200/1200. As a result of the treatment, the supportability of both lower extremities was achieved and the "duck gait" was eliminated. The child walked with a barely noticeable limp. Trendelenburg's symptom on both sides is negative. 
In the hip joints, the movements reached a satisfactory volume. On the plain X-ray of the pelvis: the reconstructed proximal ends of the femurs are located in the depressions, the cervico-diaphyseal angle is 1200 , and the former head and the "elongated" neck are centered in the acetabulum.

\section{Results and Discussion}

The results of treatment were analyzed over 8 months to 7 years. Clinical outcomes were assessed by McKey. Treatment outcomes were assessed according to the achievement of anatomical and functional outcomes. Operations of open reduction of the head and stump of the femoral neck were reduced to opening the hip joint, revision of the proximal end of the femur and the cavity, the obligatory removal of the interpolate formed from the scar tissue, and intertrochanteric shortening detorsion-varizing-"lengthening" and economical cutting of the osteotomy. With the growth of children, the lateralization of the femur gradually increased, which contributed to the restoration of the function of the gluteus medius and small muscles (Agarwal \& Rastogi, 2021; Agarwal \& Gupta, 2012). Achieved movement in the joint within the normal range of 800 , abduction remained slightly limited, but we did not notice leading contractures in the long term. In all patients, lameness and internal rotation of the lower extremities disappeared when walking, and the range of motion in the hip joint increased. Improved radiometric indices characterizing the ratio of the acetabulum and femoral head, femoral head and neck, greater trochanter. The centralization of the stump of the head and neck of the femur in the cavity improved, the SDA was 1150-1200; UV$20^{\circ} \pm 4,2$.

\section{Conclusion}

After hematogenous osteomyelitis of the proximal end of the femur, destruction of the head and neck of the femur is often observed, up to their destruction. The optimal age for surgical treatment of pathological dislocation of the hip, according to our data, is 4-5 years of age of the child, because by this time the process of ossification of the structures of the hip joint ends in most patients, and early surgical intervention often causes severe secondary deformities, up to their destruction (Boland Jr, 1972; Harrington et al., 2007). According to our data, indications for one or type of intra-articular surgery should be set strictly individually for each patient, depending on the patient's age, the type and degree of deformation of the elements of the hip joint, and the magnitude of the shortening of the lower limb. In the surgical treatment of pathological dislocation, the primary task is to restore the support function of the limb while maintaining mobility in the joint, followed by solving the problems of eliminating secondary deformities and equalizing the length of the lower limbs. Our observations indicate that open reduction of the stump of the head and neck of the femur with intertrochanteric shortening detorsion-valgus and "lengthening" neck osteotomy in most patients eliminate flexion-adduction and external-rotational contractures, provide stability of the hip joint and thereby improve gait and statics of the patient, reduce the skew of the pelvis and eliminate the Trendelenburg symptom (Stief et al., 2011; Mueske et al., 2019). The restoration of the range of motion in the hip joint depends on the degree of damage to the head and neck of the femur.

\section{References}

Agarwal, A., \& Gupta, N. (2012). Risk factors and diagnosis of developmental dysplasia of hip in children. Journal of clinical Orthopaedics and Trauma, 3(1), 10-14. https://doi.org/10.1016/j.jcot.2011.11.001

Agarwal, A., \& Rastogi, P. (2021). Outcome of acute septic dislocation of hip in children reduced at arthrotomy. Journal of Clinical Orthopaedics and Trauma, 13, 95-98. https://doi.org/10.1016/j.jcot.2020.12.007

Akzhigitov, G. N. (1998). Yudin Ya. B. Haematogenic osteomyelitis. M: Medicina.

Boland Jr, A. L. (1972). Acute hematogenous osteomyelitis. Orthopedic Clinics of North America, 3(1), 225-239. https://doi.org/10.1016/S0030-5898(20)32191-X

Cheng, J. C., Aguilar, J., \& Leung, P. C. (1995). Hip reconstruction for femoral head loss from septic arthritis in children. A preliminary report. Clinical orthopaedics and related research, (314), 214-224.

Choi, I. H., Pizzutillo, P. D., Bowen, J. R., Dragann, R., \& Malhis, T. (1990). Sequelae and reconstruction after septic arthritis of the hip in infants. JBJS, 72(8), 1150-1165.

Di Gennaro, G. L., Stagni, C., Magnani, M., Libri, R., \& Donzelli, O. (2003). Trochanteric arthroplasty in the treatment of sequelae after septic arthritis of the hip in infancy. La Chirurgia degli organi di movimento, 88(4), 377-384.

Forlin, E., \& Milani, C. (2008). Sequelae of septic arthritis of the hip in children: a new classification and a review of 41 hips. Journal of Pediatric Orthopaedics, 28(5), 524-528. 
Garkavenko, Y. E., \& Pozdeev, A. P. (2013). Orthopedic Aid To Children With Sequelae Of Hematogenous Osteomyelitis Of Long Bones In The Turner Orthopedic Institute. Pediatric Traumatology, Orthopaedics and Reconstructive Surgery, 1(1), 16-20.

Garkavenko, Yu.E., \& Pozdeev, A.P. (2011). Orthopedic consequences of hematogenous osteomyelitis of long bones in children (clinical picture, diagnosis, treatment). SPb .: FGBU NIDOI im. GI Turner .

Harrington, M. E., Zavatsky, A. B., Lawson, S. E. M., Yuan, Z., \& Theologis, T. N. (2007). Prediction of the hip joint centre in adults, children, and patients with cerebral palsy based on magnetic resonance imaging. Journal of biomechanics, 40(3), 595-602. https://doi.org/10.1016/j.jbiomech.2006.02.003

Kardina, I., Ramadany, S., Sanusi B, Y., Made, S., Stang, S., \& Syarif, S. (2020). Children's midwifery learning media application about android-based rough motor development in improving midwifery student skills. International Journal of Health \& Medical Sciences, 3(1), 146-152. https://doi.org/10.31295/ijhms.v3n1.300

Karmazyn, B. (2010, April). Imaging approach to acute hematogenous osteomyelitis in children: an update. In Seminars in Ultrasound, $\quad C T$ and MRI (Vol. 31, No. 2, pp. 100-106). WB Saunders. https://doi.org/10.1053/j.sult.2009.12.002

Korovin, S. A., Dzyadchik, A. V., Dvovenko, E. V., \& Sokolov, Yu. Yu. (2018). Laparoscopic interventions in children with complicated appendicitis. Medical advice, (17).

Krys'-Pugach, A. P. (2008), guk ju. N., Kutsenok ya. B., Darovskii AS Our experience in surgical treatment of orthopedic consequences of purulent coxitis in children. Travma. Trauma, 1, 66-70.

Labuzov, D.S., Salopenkova, A.B., \& Proshchenko, Ya.N. (2017). Methods for the diagnosis of acute epiphyseal osteomyelitis in children. Pediatric Traumatology, Orthopedics and Reconstructive Surgery, 5 (2).

Lew, D. P., \& Waldvogel, F. A. (2004). Osteomyelitis. The Lancet, 364(9431), 369-379. https://doi.org/10.1016/S0140-6736(04)16727-5

Morrey, B. F., \& Peterson, H. A. (1975). Hematogenous pyogenic osteomyelitis in children. Orthopedic Clinics of North America, 6(4), 935-951. https://doi.org/10.1016/S0030-5898(20)30956-1

Mueske, N. M., Õunpuu, S., Ryan, D. D., Healy, B. S., Thomson, J., Choi, P., \& Wren, T. A. (2019). Impact of gait analysis on pathology identification and surgical recommendations in children with spina bifida. Gait \& posture, 67, 128-132. https://doi.org/10.1016/j.gaitpost.2018.10.003

Nikolaev A.I. (2005) Prevention and earlier complex treatment of children with pathological dislocation of the hip: Abstract of the ... Candidate of Medical Sciences: - St. Petersburg. - p. 16.

Parsons, B., \& Strauss, E. (2004). Surgical management of chronic osteomyelitis. The American journal of surgery, 188(1), 57-66. https://doi.org/10.1016/S0002-9610(03)00292-7

Shamsiev, Zh.A., Shamsiev, A.M., \& Makhmudov, Z.M. (2018). On the issue of early diagnosis of acute hematogenous osteomyelitis of the bones of the hip joint in children. Pediatric Surgery, 22 (2).

Shikhabudinova P.A., Makhachev S.M., Yakhyaev Ya.M., Askerov A.A. (2000) Analysis of conservative and surgical treatment of pathological dislocation of the femoral head in young children. Pediatric surgery. No3. $-\mathrm{p}$. 53

Sitkovsky, N. B., \& Kukuruza, Y. P. (1975). Affection of the hip joint in hematogenous osteomyelitis in children (Russian). Клінічна хірургія, (10), 1-7.

Skvortsov, A. P., Gil'mutdinov, M. R., Akhtyamov, I. F., Skvortsov, A. P., Gil'mutdinov, M. R., Gil'mutdinov, M. R., \& Akhtyamov, I. F. (2011). Prevention of Orthopaedic Consequences in Hip Joint Area in Children after Epimetaphyseal Osteomyelitis. NN Priorov Journal of Traumatology and Orthopedics, 18(3), 44-47.

Sokolovsky, A. M., \& Sokolovsky, O. A. (1997). Pathological hip dislocation.

Stief, F., Böhm, H., Schwirtz, A., Dussa, C. U., \& Döderlein, L. (2011). Dynamic loading of the knee and hip joint and compensatory strategies in children and adolescents with varus malalignment. Gait \& posture, 33(3), 490495. https://doi.org/10.1016/j.gaitpost.2011.01.001

Suwananta, I. M., Ariawati, K., Widnyana, A. A. N. K. P., \& Lastariana, K. A. Y. (2021). Prevalence and characteristic of pediatric solid tumor in Sanglah Hospital Bali. International Journal of Health \& Medical Sciences, 4(3), 322-332. https://doi.org/10.31295/ijhms.v4n3.1755

Teplenky, M.P., Dyachkova, G.V., Oleinikov, E.V., \& Bunov, V.S. (2016). Comparative characteristics of radiological classifications of septic coxitis consequences in children. The genius of orthopedics, (4). 